\title{
The Costume of Wuji in the Dunhuang Murals of Tang Dynasty
}

\author{
Yiliang $\mathrm{Jia}^{1}$ \& Wenjuan $\mathrm{Ma}^{2}$ \\ ${ }^{1}$ Fashion, Art Design Institute, Donghua University, Shanghai, China \\ ${ }^{2}$ Donghua University Press, Shanghai, China \\ Correspondence: Yiliang Jia, Fashion, Art Design Institute, Donghua University, West Yanan Road, 1882\#, \\ Shanghai, China. Tel: 86-138-1712-7318. E-mail: jyldhdh@gmail.com
}

Received: September 5, 2012 Accepted: October 17, 2012 Online Published: December 31, 2012

doi:10.5539/ass.v9n1p299 URL: http://dx.doi.org/10.5539/ass.v9n1p299

\begin{abstract}
According to the statistic, there're total 8 basic costume styles of WUJI in the Dunhuang Murals of Tang Dynasty. This paper analyses the 110 dancers' image, investigates the literature, have the image restoration and compare it with the related characters, all of which makes up the shortcomings of doing research only by mutual proving of images and literature. The static dancing posture will no longer be the only criteria to judge the dancing style and its artistic origins.
\end{abstract}

Keywords: Tang dynasty, Wuji, costume

\section{Introduction}

According to the statistics released by the music and dance laboratory of DunHuang Research Institute, there're over 200 grottoes having dance and music images in Mogao Grottoes, in which the majority of over 3400 dance and music drawing bodies are Yueji. Due to the characteristic of 'No difference between Music and Dance' in China's traditional music and dance culture, the appellation of "Wuji" is extremely rare. This paper only introduces the entertainer who primarily plays the musical instrument "Yueji" and the artiest who primarily dance "Wuji".

\section{General Situation of Wuji Costume}

Based on the analysis of more than one hundred costumes of Wuji in Tang Dynasty, we will have a preliminary understanding of WUJI costume style of Dunhuang in Tang Dynasty Jingbianhua. Except head and baldric (the style of Wuji's head is basically the same; we can refer to papers of YeJing and LiMin etc. Baldric is mainly consisted of YingLuo, arm bracelet, bracelets and long scarf ribbon. The basic style is also very similar with only some differences in details. This paper will not discuss more about that), according to the statistics, there're totally 8 basic costume styles of Wuji body presented. In accordance with the principle of bare skin level, the top-down and out-inside is as follows:

\subsection{The Naked Upper Body}
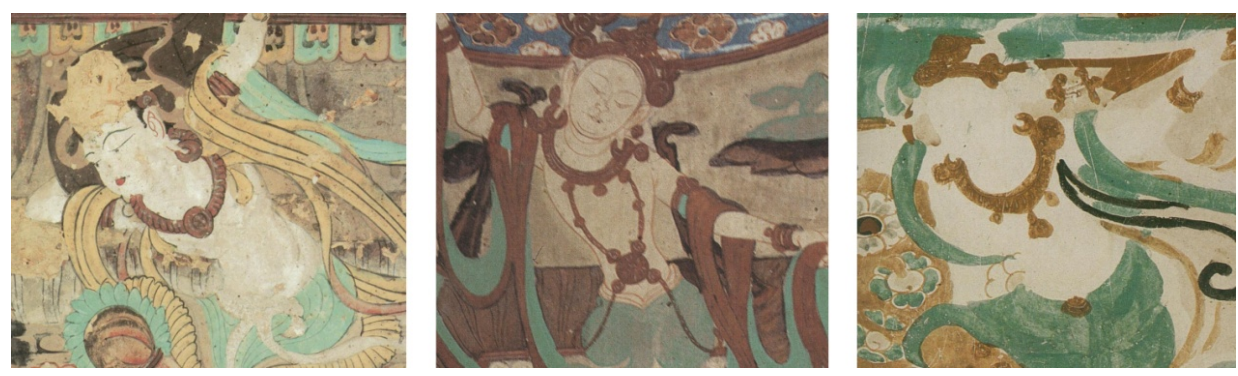

Figure 1.

It's the costume without upper, just decorated with YingLuo, hand grasp or shoulder ribbon in neck. Because Luocheng is very common in Dunhuang murals and is a composition of WUJI costume, it's regarded as one of the basic styles. 


\subsection{Luoye}
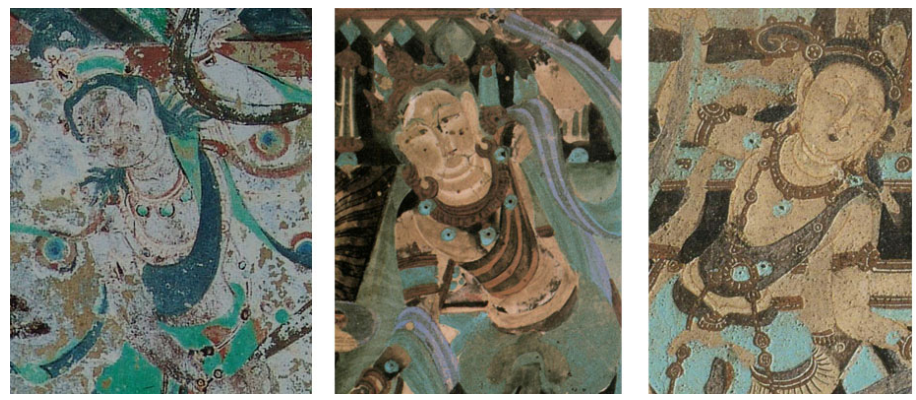

Figure 2.

It's the rectangle scarf ribbon which extends from the right oxter around breast to the left shoulder and is fixed by twining or knotting. Majorities are two-sided and heterochrome, with red, blue or green for common. It is conjectured by the dressing effect that the ribbon should be $200 \mathrm{~cm}-300 \mathrm{~cm}$ long and $40-80 \mathrm{~cm}$ wide and the shell fabric is thin and soft. Usually, there's no fixed pattern on the chest and the tail end is separated by two parts which are fluttered from oxters. The pattern of draping and surrounding can not be explained in a conventional way. The naming by Scholars is different, such as heaven clothes, tussores colthes, brocade scarf, brocade shawl, oblique shawl, silk shawl, silk Pei etc., so many different names are defined from different points of views.

\subsection{The Wide Plait Collar}

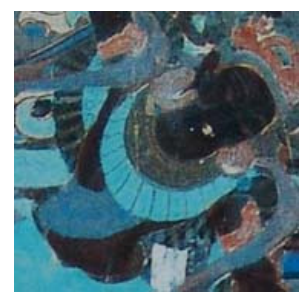

Figure 3.

The wide plait collar, mostly colored in green, blue and cyan, is annular after being unfolded. Because it is used to cover the chest, it's conjectured that the radius is around $30 \mathrm{~cm}$. The collar is made of the precious pearl and YingLuo ring which is tough, stiff and smooth; in addition to being the independent tops, it is usually represented through multi-layers superposition or being connected, and collocated with LUOYE or tight clothes in the mural.

\subsection{Tight Clothes}
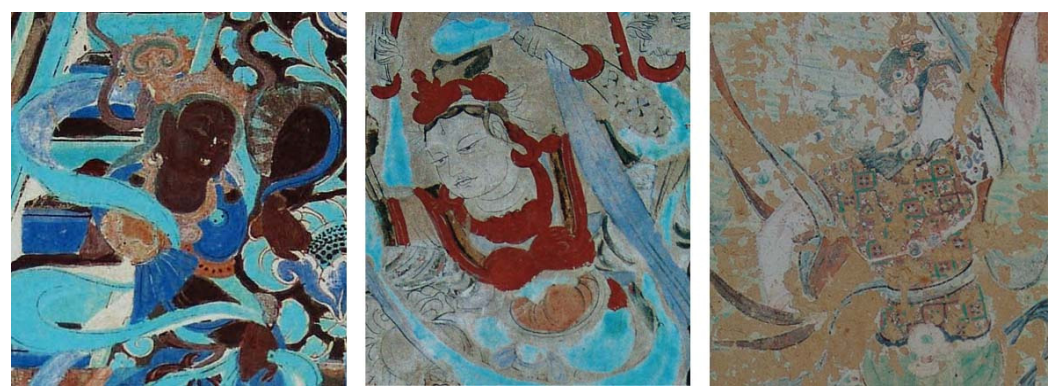

Figure 4.

These are the tight and fitted tops. WUJI's tight clothes are mostly colored in red or dark with the thick and non-transparent shell fabric. The styles are almost the same with some differences in collar, sleeve and sweep. Majorities of sleeves are short and long ones. The elbow is decorated by wide plait. Long sleeve cuff is consisted of close and unclose. Sweep is consisted of four sorts, namely, To under the chest, To waistline, splayed edging and To skirt. Tight clothes are usually fastened by the ribbon at waist to emphasize the smart activity of waist. The waist ribbon has several long, short, wide and narrow states. The tail end of the long waist ribbon can wave along with dancing. Ribbon can be replaced by YINGLUO bracelets sometimes. 


\subsection{Waist Skirt}
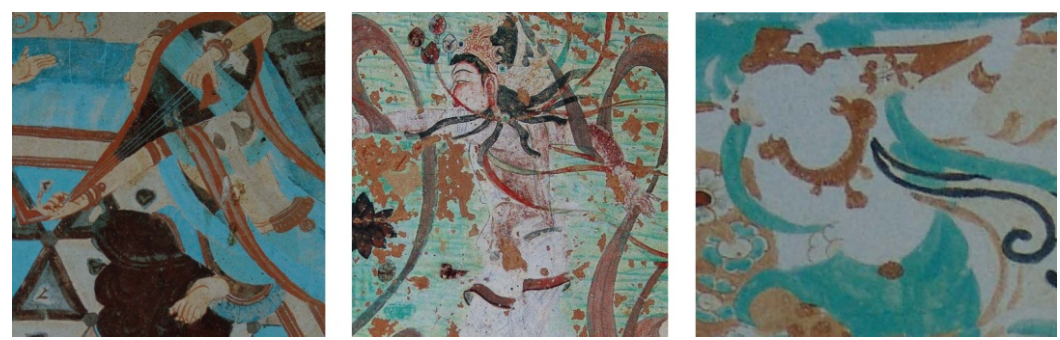

Figure 5. Long pants

Although there's a 'waist' in the name of waist skirt, it is a broad shawl actually which is tied on crotch, fixed or knotted by jewelry in front of belly. As the most popular and basic pattern of WUJI costume, waist skirt almost appears at every WUJI. Mostly, it is pure color (the majority is green and occasionally, it is cyan and blue) in rarely patterns, soft and non-transparent. It's usually as long as just covering the hip, occasionally to knee. Although the style is simple, it presents the abundant wearing effect: by means of various patterns of compassing and knotting, forms abundant drapes in front of belly, the sweep forms two beautiful curves. The change of tail end is various as well, some naturally flapping down and dancing in the wind and some symmetrical curling like RUYI head which is strongly decorated. We can say that the waist skirt is the base of WUJI's depth appearance from overlapping wearing collocation.

\subsection{Short Skirt}
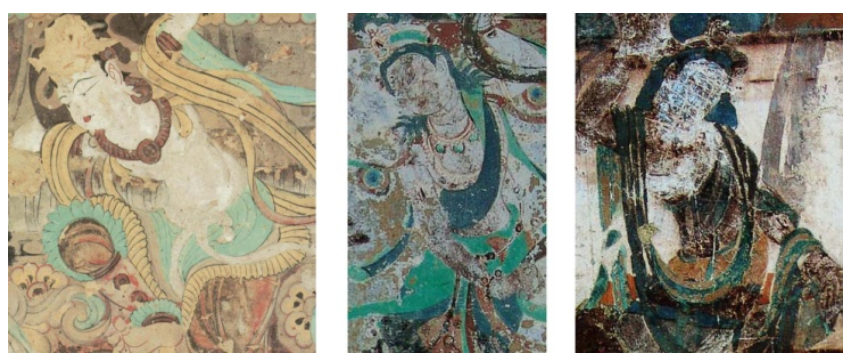

Figure 6.

The knee-length short skirt mostly matches the sweep skirt. It shall be sewn piece together, but not knotted around. Because it is worn under the waist skirt, the part of reveal usually can only be seen. The majority have the wide decorated edge, drew by crisp grass. Short skirt usually contrasts to waist skirt of different colors and forms abundant costume appearance. It's mostly blue. The shell fabric is thick and textured.

\subsection{Long Skirt}

The basic style of long skirt of WUJI is fitting the body with no parting. The length can be to ankle or ground with smooth patterns. It's mostly red outside and green inside, decorated by BAOXIANG flower pattern. The shell fabric includes non-transparent and good effect of flap down, and transparent thin material made of yarn. Its elegant smart apropos reflects the lightness of dance and wonderfulness. In the murals, when WUJI is dancing with both feet, they mostly wear long skirt. As for this style of costume, it is also named from different points of views, such as pomegranate skirt, brocade skirt, silk skirt, jacket skirt, and so on. There're a lot of monk and laic women of Dunhuang art wearing long skirt, especially the Bodhisattvas.

\subsection{Broad Foot Pants}

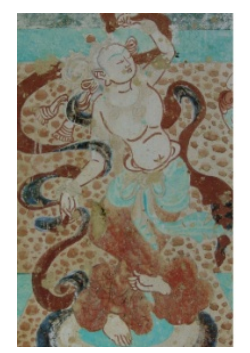

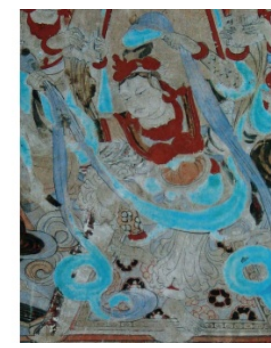

Figure 7.

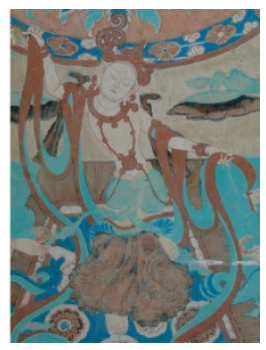


These are the broad pants which look like skirt. They can be classified into three sorts by shape approximately: 1, the length is to ankle or ground in the dark red or dark color mostly. The whole pants are broad with the complicated drape like the turn-up cloud. The texture is thick and non-transparent. 2, the length is to ankle or ground. They are mostly red outside and green inside and broaden below the knees in the shape of current flares, pants cuff's lines twists and turns. The texture is thinner and heavily flapped down. 3, the length is slightly longer than knees in the brown color mostly. They are knotted by ribbon at knees, or fixed by YINGLUO precious pearl, forms broad and flexible drape. The part above knees is loose knotted and other parts under knees are irregular flapped down. Pants cuff is decorated by blue and green. The shell fabric is stiff and smooth. Bunch leg pants are usually worn inside broad pants to form the appearance effects of abundant layers.

\section{Discussion about Color}

According to the occurrences of colors of the costumes of WUJI on the mural, the types and ranges of colors can be gotten. For example, in spite of the alternatives of colors, the long skirts are in red basically.

Table 1.

\begin{tabular}{|c|c|c|c|c|}
\hline $\begin{array}{l}\text { COLOR } \\
\text { Name }\end{array}$ & COLOR & CAVE NO. & Serial No. & Position \\
\hline white & & $205 / 172 / 112 / 237 / 360$ & $1 / 2 / 27 / 28 / 40 / 57 / 64$ & $\begin{array}{l}\text { Tight Clothes } \\
\text { Waist skirt } \\
\text { Short skirt } \\
\text { Long skirt }\end{array}$ \\
\hline red & & $\begin{array}{l}205 / 341 / 180 / 217 / 445 / 112 / \\
154 / 158 / 159 / 197 / 231 / 237 / \\
286 / 358 / 359 / 360 / 386 / 12 / \\
14 / 20 / 85 / 7108 / 156 / 196\end{array}$ & $\begin{array}{l}1 / 15 / 29 / 33 / 37 / 38 / 39 / 40 / \\
41 / 42 / 43 / 44 / 45 / 46 / 47 / 52 \\
/ 55 / 57 / 58 / 60 / 61 / 62 / 63 / 65 \\
/ 67 / 69 / 70 / 71 / 72 / 74 / 76 / 78 \\
/ 79 / 80 / 81 / 83 / 84 / 85 / 86 / 96 / \\
97 / 98 / 101\end{array}$ & $\begin{array}{l}\text { LUOYE } \\
\text { Tight Clothes } \\
\text { Waist skirt } \\
\text { Short skirt/Long skirt } \\
\text { Broad Foot Pants } \\
\text { Bunch Leg Pants } \\
\text { Fold trim }\end{array}$ \\
\hline green & & $\begin{array}{l}205 / 220 / 335 / 341 / 45 / 148 / \\
217 / 445 / 154 / 158 / 159 / 237 / \\
286 / 358 / 359 / 360 / 386 / 12 / \\
20 / 85 / 156 / 196 / 361 / 370 \\
148 / 172 / 180 / 217 / 320 / 112 / \\
154 / 186 / 197 / 200 / 201 / 231 / \\
359 / 386 / 12 / 14 / 18 / 108 / \\
156 / 361 / 486\end{array}$ & $\begin{array}{l}1 / 2 / 3 / 5 / 7 / 13 / 1516 / 17 / 25 / \\
33 / 34 / 3742 / 44 / 45 / 46 / 47 / \\
58 / 59 / 60 / 62 / 64 / 65 / 66 / 69 / \\
74 / 76 / 77 / 79 / 80 / 82 / 93 / \\
101 / 105 / 10623 / 24 / 25 / 27 / \\
29 / 34 / 35 / 36 / 38 / 39 / 41 / 43 / \\
50 / 52 / 53 / 54 / 55 / 61 / 62 / 66 / \\
70 / 71 / 72 / 73 / 84 / 85 / 92 / 94 / \\
95 / 96 / 97 / 98 / 99 / 103 / 107\end{array}$ & $\begin{array}{l}\text { Wide plait collar } \\
\text { LUOYE } \\
\text { Tight Clothes } \\
\text { Waist skirt } \\
\text { Short skirt } \\
\text { Bunch Leg Pants } \\
\text { Fold trim }\end{array}$ \\
\hline blue & & $\begin{array}{l}220 / 148 / 217 / 159 / 358 / \\
359 / 360 / 396 / 156\end{array}$ & $\begin{array}{l}3 / 4 / 5 / 23 / 24 / 25 / 34 / \\
46 / 48 / 95 / 62 / 63 / 65 / 97\end{array}$ & $\begin{array}{l}\text { Wide plait collar } \\
\text { LUOYE }\end{array}$ \\
\hline
\end{tabular}




\begin{tabular}{|c|c|c|c|}
\hline & & & Tight Clothes \\
\hline & & & Short skirt \\
\hline & & & Bunch Leg Pants \\
\hline & & & Fold trim \\
\hline \multirow[t]{2}{*}{ purple } & $231 / 358 / 12$ & $55 / 59 / 70$ & U-W Yinluo \\
\hline & & & Fold trim \\
\hline yellow & 112 & $40 / 41$ & Fold trim \\
\hline \multirow[t]{4}{*}{ brown } & $360 / 18 / 156 / 370$ & 63/73/90/91/92/93/94 & LUOYE \\
\hline & & /95/99/106 & Tight Clothes \\
\hline & & & Short skirt \\
\hline & & & Broad Foot Pants \\
\hline \multirow[t]{6}{*}{ black } & 205/220/335/45/148/ & $1 / 7 / 8 / 13 / 16 / 23 / 25 / 26 / 27 / 28 /$ & Tight Clothes \\
\hline & $172 / 180 / 320 / 158 / 159 /$ & $29 / 35 / 36 / 45 / 46 / 48 / 53 / 54 / 55 /$ & Short skirt \\
\hline & $200 / 231 / 237 / 286 / 358 /$ & $57 / 58 / 59 / 60 / 61 / 62 / 64 / 66 / 69 /$ & Long skirt \\
\hline & $360 / 38612 / 85 / 156 / 196$ & 70/71/72/73/77/80/82/90/91/ & Broad Foot Pants \\
\hline & $/ 361 / 370$ & 92/93/9495/96/97/98/99/100/ & Bunch Leg Pants \\
\hline & & 103/104/105/106 & \\
\hline
\end{tabular}

\section{Discussion about Patterns}

BAOXIANG pattern is the most common in the costumes of WUJI and it can be divided into four petals of cross shape found in grotto 220 and 66 , as well as the composite type with multi-layer and dense petals found in grotto 360 and 108, etc. These two patterns were transformed from lotus pattern, indicating the stateliness of Buddhism.

Table 2.

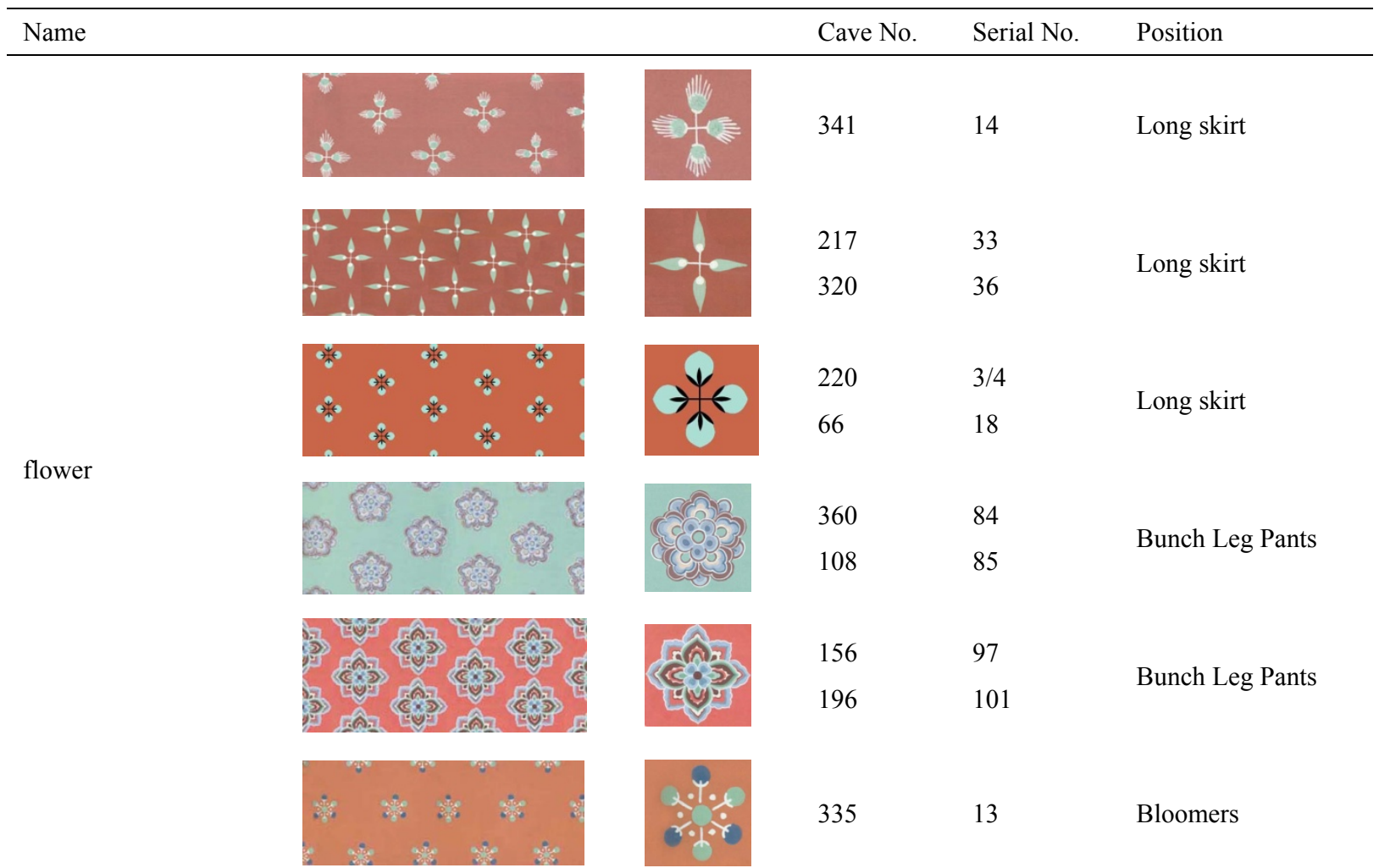



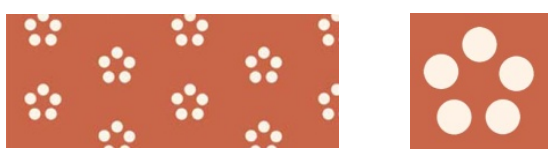

205

$1 / 2$

Long skirt
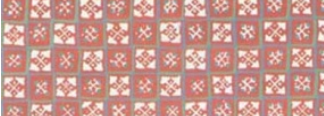

Checkerboard

Volume grass grain
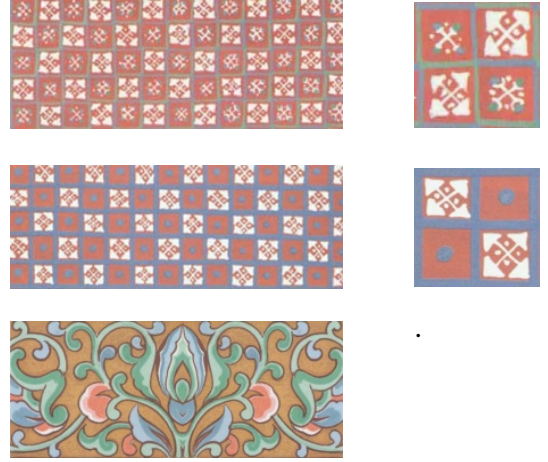

220

4

Tight Clothes
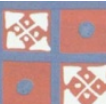

220

3

Tight Clothes

220

5

Short skirt

12

Bunch Leg Pants

Else

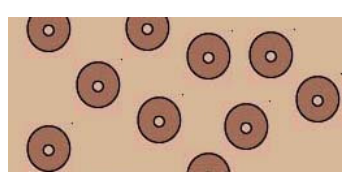

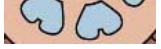

12
70

Bunch Leg Pants

\section{Matching Skills}

The costumes with lines can be matched together. What's more, there are different details in each of the basic style, so the costumes are ever-changing and varied. In the past, the researchers and scholars usually divided the dance into "JIANWU" and "RUANWU" according to the moves of dance. In this way, the styles of the costumes of WUJI can also be split into "soft" style and "vigorous" style.

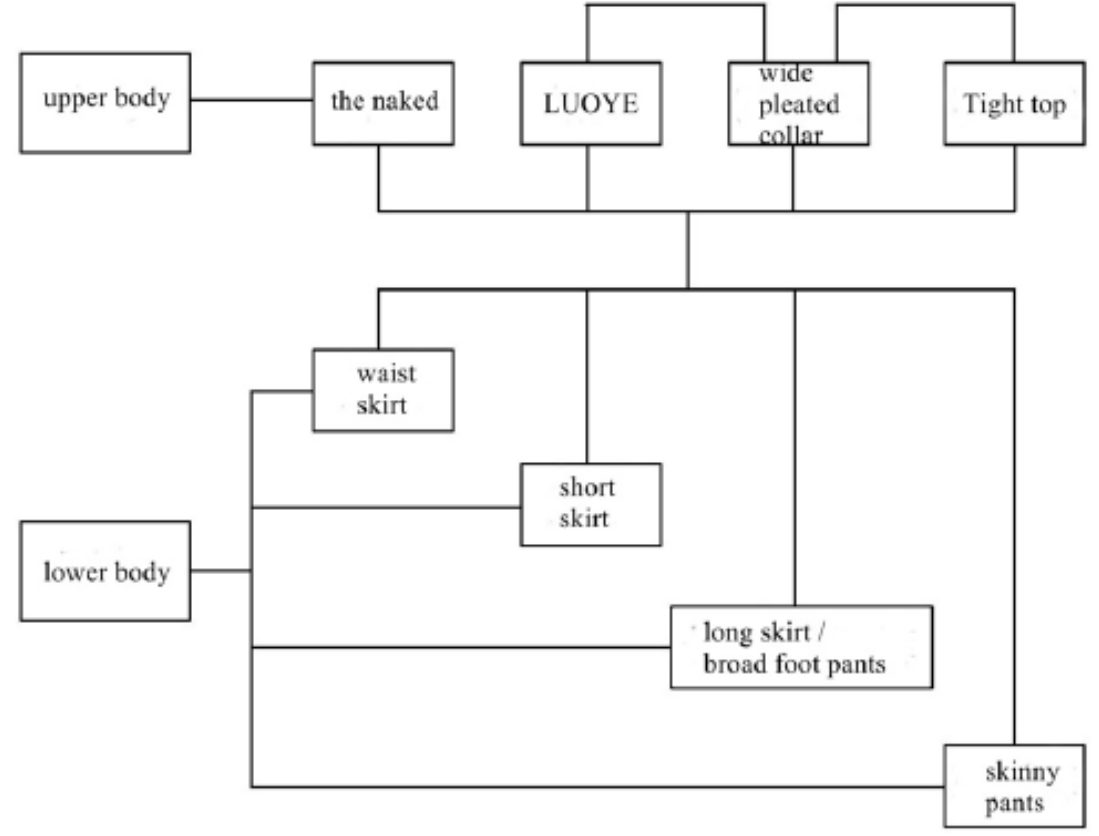

Figure 8 .

\section{1 "Soft" Style}

Basic style of matching: the naked upper body / LUOYE+ long skirt

In such a dressing style, the long skirt is typical. It is obvious that the costumes of WUJI in "RUANWU" are "soft" to a certain degree and such a style is positively correlated with the bareness degree of the WUJI. 


\section{2 "Vigorous" Style}

Basic style of matching: Tight top+ skinny pants

If there are either the tight top or skinny pants, the costumes can be regarded as the "vigorous" style. The tight top with skinny pants will wrap up the body of the WUJI and highlight the dynamic dance moves. Apparently, the costumes of WUJI in "JIANWU" tend to be "vigorous".

\subsection{Costumes between the Styles Mentioned Above}

Basic style: wide pleated collar, waist skirt, short skirt and broad foot pants.

Such costumes are neutral and auxiliary without unique styles.

\section{Conclusion}

Through the analysis on the eight costumes, matching skills, colors and patterns, it can be found that the costumes of WUJI in Buddhist ceremony are closely related to the dance. The costumes are designed to reflect the dynamic dance and highlight the body movement. The analysis on the styles of costumes and dance moves will help to further define the types of dance and facilitate the related studies.

\section{Acknowledgments}

The paper's author wishes to thank Mr. Yuan Zheng for his help and efforts in completing this research.

\section{References}

Duan, W. J. (1991). China Mural Collections. Mural of Grotto. Tianjin: Tianjin people's art press.

Rao, Z. Y. (1986). Dunhuang Songs, dances, and Qiuci songs. Xinjiang Art.

Wang, K. F. (1987). The History of Chinese Dance the Sui, Tang and Five Dynasties Part. Beijing: Beijing Culture Art Press.

Wang, K. F. (2001). Dance painting scroll. Hong Kong: Commercial Press. 